\title{
Blinde Justitia?
}

\author{
Die Entdeckung des Missbrauchs in der Rechtspraxis
}

\author{
Frauke Rostalski
}

\section{Einleitung}

Sexueller Missbrauch von Kindern gehört zum Kreis der schwerwiegendsten Straftaten, die unser Rechtssystem kennt. Erschütternde Fälle der jüngeren Vergangenheit ${ }^{1}$ haben dazu geführt, dass diesem Gewicht des Unrechts sexualisierter Gewalt gegen Kinder ${ }^{2}$ auch die Strafrahmen der einschlägigen Vorschriften des deutschen Strafgesetzbuchs künftig besser Rechnung tragen sollen. ${ }^{3}$ Beispielhaft soll der Grundtatbestand der sexualisierten Gewalt gegen Kinder künftig als Verbrechen mit einem Mindeststrafmaß von einem Jahr Freiheitsstrafe ausgestaltet sein und im Höchstmaß mit 15 Jahren Freiheitsstrafe geahndet werden (bisher handelte es sich um ein Vergehen mit Freiheitsstrafe von sechs Monaten bis zu zehn Jahren). ${ }^{4}$ Kinder (Personen unter 14 Jahren) und Jugendliche sind in ihrer sexuellen Selbstbestimmung besonders

1 Aufsehen erregten vor allem der hundertfache Kindesmissbrauch auf einem Campingplatz im westfälischen Lügde, siehe https://www.zeit.de/gesellschaft/zeitgeschehen/2019-o6/ luegde-hundertfacher-kindesmissbrauch-angeklagten-gestaendnis-prozessauftakt, und ein in Münster aufgedecktes bundesweites Missbrauchs-Netzwerk, siehe https://www.tagesschau.de/inland/razzien-festnahmen-101.html. Zuletzt wurden im Zuge der Ermittlungen im Missbrauchskomplex Bergisch-Gladbach über 3 o ooo Datenspuren gefunden, die mittlerweile zu 200 namentlich identifizierten Tatverdächtigen geführt haben, siehe https:// www.zeit.de/gesellschaft/zeitgeschehen/2020-10/zwoelf-jahre-haft-fuer-angeklagtenim-missbrauchsfall-bergisch-gladbach; https://www.tagesschau.de/investigativ/zapp/ kindesmissbrauch-bergisch-gladbach-1o3.html. Alle in diesem Beitrag genannten Internetadressen wurden, sofern nicht anders angegeben, zuletzt aufgerufen am 14.12.202O.

2 Die bisher im StGB verwendete Bezeichnung "Sexueller Missbrauch von Kindern“ in den $§ \S 176-176$ b soll im Gesetzesentwurf der Bundesregierung (RegE) durch den Begriff der „Sexualisierten Gewalt gegen Kinder" ersetzt werden, siehe RegE: Entwurf eines Gesetzes zur Bekämpfung sexualisierter Gewalt gegen Kinder vom 21.10.2020, einzusehen unter https:// www.bmjv.de/SharedDocs/Gesetzgebungsverfahren/Dokumente/RegE_Bekaempfung_sex_ Gewalt_Kinder.pdf?_blob=publicationFile\&v=2, 1 und 5 ff. Die Änderung soll dem begrifflichen Missverständnis vorbeugen, dass es auch einen erlaubten „Gebrauch“ von Kindern gebe, siehe BMJV: Reformpaket zur Bekämpfung sexualisierter Gewalt gegen Kinder vom 1.7.2020, einzusehen unter https://www.bmjv.de/SharedDocs/Downloads/DE/News/ Artikel/o10720_Reformpaket_Missbrauch.pdf?_blob=publicationFile\&v=1, 1.

3 Zu Änderungen der materiellen Rechtslage auf Tatbestandsebene siehe RegE (Fn. 2), $5 \mathrm{ff}$.

4 Zu weiteren Modifikationen der bisherigen Rechtslage siehe ebd., 1off. 
schutzwürdig. Erfahrungen sexualisierter Gewalt können erhebliche Spuren in der kindlichen Seele hinterlassen, die nicht selten ein Leben lang als schmerzhafte Narben verbleiben.

Es liegt damit auf der Hand, welch hohe Bedeutung der Prävention in diesem Bereich zukommt. Wichtig ist, dass es erst gar nicht zu sexuellen Übergriffen auf Kinder kommt. Sofern dies gleichwohl geschieht, erlangt das staatliche Strafrecht eine hervorgehobene Rolle. Strafe dient nicht der Prävention neuer Taten. ${ }^{5}$ Sie dient aber der Wiederherstellung des durch die Tat gestörten Rechtsfriedens und dabei auch dem Opfer, das mit seiner Leiderfahrung nicht allein gelassen wird. Durch die angemessene strafende Reaktion auf das Fehlverhalten des Täters bedeutet die Gesellschaft gegenüber dem einzelnen Opfer zugleich, dass das ihm widerfahrene Unrecht nicht geduldet wird. Es handelt sich hierbei nicht um eine Belanglosigkeit. Im Gegenteil kann es für das Opfer eine ganz erhebliche Bedeutung haben, dass auch die Gesellschaft dem Täter entgegentritt und sein Verhalten verurteilt. Auf diese Weise können Verletzungen nicht ungeschehen gemacht werden. Dies ist aber auch nicht Aufgabe des Strafrechts. Vielmehr geht es darum, dem Täter infolge seiner Straftat entschieden entgegenzutreten, indem ihm mitgeteilt wird, dass sein Verhalten falsch war und seitens der übrigen Gesellschaftsmitglieder verurteilt wird. Dieser Akt der Kommunikation mit dem Täter erfüllt gerade auch eine hohe Bedeutung gegenüber dem Opfer, das sich in seinen Interessen gesehen und geschätzt fühlt - die an ihm vorgenommene Straftat erfüllt Unrecht, weil seine persönlichen Interessen beeinträchtigt wurden. Auf diese Weise dient die Bestrafung auch dem Opfer.

Nicht zuletzt vor diesem Hintergrund wiegt ein staatliches Unterlassen gebotener Strafverfolgung besonders schwer. Bleibt die Gesellschaft infolge der Begehung erheblichen Unrechts stumm, sendet dies auch an das Opfer der Tat ein bitteres Signal: Es steht - bezogen auf die staatliche Gemeinschaft verkörpert durch die Strafrechtspflege - mit seinem erlittenen Leid allein. Weil es nicht zur Bestrafung kommt, bleibt die Straftat von Rechts wegen unwidersprochen. Von nicht wenigen dürfte dies als Form der sekundären Viktimisierung empfunden werden - also als erneute Vertiefung der Erfahrung, Opfer geworden zu sein, indem nämlich die eigenen, berechtigten Interessen übersehen werden. ${ }^{6}$

5 Zur umfangreichen Begründung siehe Frauke Rostalski, Der Tatbegriff im Strafrecht. Tübingen 2019, $36 \mathrm{ff}$.

6 In einer im Jahr 2001 veröffentlichten US-amerikanischen Studie zur sekundären Viktimisierung von vergewaltigten Frauen wurde der Kontakt zur Strafverfolgungsbehörde vor allem dann als erneute Verletzung empfunden, wenn die Tat im Nachgang nicht strafrechtlich verfolgt wurde, siehe Campbell u.a., Journal of interpersonal Violence 16, 2001, 1239 
Straftaten finden in sämtlichen gesellschaftlichen Bereichen statt. Die Kirche stellt hiervon keine Ausnahme dar. Dennoch hat das Ausmaß sexualisierter Gewalt gegen Kinder und Jugendliche durch Vertreter der katholischen Kirche, wie es in den letzten Jahren und insbesondere durch die MHG-Studie ${ }^{7} 2018$ in der Öffentlichkeit bekannt geworden ist, viele erschüttert. In meinem Beitrag befasse ich mich damit, wie von Seiten deutscher Strafverfolgungsbehörden hierauf bis heute reagiert wurde. Ich möchte mich dabei in einen Diskurs begeben mit einigen ihrer Vertreter, die sich hierzu etwa in Presseberichten oder durch amtliche Beschlüsse geäußert haben, um so die Gründe näher zu untersuchen, die für oder gegen die Aufnahme staatlicher Ermittlungen und potentielle Verurteilungen sprechen.

\section{Ausmaß des sexuellen Missbrauchs von Kindern und Jugendlichen in der katholischen Kirche}

Wir blicken im Jahr 2020 auf zehn Jahre der intensiven gesellschaftlichen Befassung mit Fällen des sexuellen Missbrauchs und den diese etwaig ermöglichenden Strukturen innerhalb der katholischen Kirche zurück. Genaue Zahlen können nach wie vor nicht geliefert werden. Mit der MHG-Studie sind uns allerdings wichtige Anhaltspunkte für die tatsächliche Verbreitung von sexualisierter Gewalt gegen Kinder und Jugendliche in den Jahren 1946 bis 2014 geliefert worden. Die Forscher haben insgesamt 3677 sexuelle Übergriffe von mindestens 1670 katholischen Priestern und Ordensleuten in diesem Zeitraum festgestellt. Die Opfer seien überwiegend männliche Minderjährige gewesen, mehr als die Hälfte von ihnen seien zum Tatzeitpunkt jünger als 14 gewesen. Bei $15 \%$ der Missbrauchsfälle soll es zu einem oralen, analen oder vaginalen Eindringen gekommen sein. ${ }^{8}$ Es ist in der Vergangenheit immer wieder auch zur Verurteilung von Priestern und anderen Kirchenangehörigen wegen Straftaten der sexualisierten Gewalt gegen Kinder gekommen. ${ }^{9}$ Fest

und 125of. Ein Überblick über bisherige Studien zur sekundären Viktimisierung findet sich etwa bei Prues/Hoffmann/Rettenberger, Monatsschrift für Kriminologie und Strafrechtsreform 102, 2019, 184 und 189f. und bei Volbert, in: Barton/Kölbel (Hrsg.) Ambivalenzen der Opferzuwendung des Strafrechts, 2012, 197 und 198ff.

7 Vgl. Dreßing u.a., Sexueller Missbrauch an Minderjährigen durch katholische Priester, Diakone und männliche Ordensangehörige im Bereich der deutschen Bischofskonferenz. Projektbericht, einzusehen unter https://www.dbk.de/fileadmin/redaktion/diverse_downloads/dossiers_2018/MHG-Studie-gesamt.pdf.

8 Ebd., 8 und 174 .

9 Das Landgericht (LG) Deggendorf etwa verurteilte im Jahr 2018 einen ehemaligen Priester wegen 108 Missbrauchstaten zu einer Freiheitsstrafe von achteinhalb Jahren und ordnete die 
steht, dass zumindest nach der Publikation der MHG-Studie Strafverfolgungsbehörden in ihrer Tätigkeit verstärkt in den Fokus der öffentlichen Wahrnehmung gerückt sind. Hierzu hat auch eine Strafanzeige des Instituts für Weltanschauungsrecht gemeinsam mit meinen Kollegen und Strafrechtsprofessoren Holm Putzke, Eric Hilgendorf, Rolf Dietrich Herzberg, Reinhard Merkel, Ulfrid Neumann und Dieter Rössner beigetragen. ${ }^{10}$ Von Bedeutung ist zudem die Aussage des MHG-Studienleiters Harald Dreßing in Bezug auf die Aussagekraft seiner Ergebnisse: „Und wir können sicher sagen: Das ist die Spitze des Eisbergs. Weniger war es sicherlich nicht. Wir wissen, es war sicherlich mehr. Denn wir wissen auch: Es sind Akten vernichtet worden, es sind auch Akten manipuliert worden." ${ }^{\text {"11 }}$ Mehrere Staatsanwaltschaften haben zwischenzeitlich Ermittlungen aufgenommen und deren (vorläufige) Ergebnisse mitgeteilt. ${ }^{12}$ Aus rechtswissenschaftlicher Perspektive bleiben dabei nicht selten viele Fragen offen.

Unterbringung in einem psychiatrischen Krankenhaus an, siehe LG Deggendorf Urteil vom 22.02.2018 - 1 KLs 4 Js 15941/16 jug, Beck-online Rechtsprechung 2018, 49819. Jüngst wurden gegen die Verurteilung eines Priesters wegen sexuellen Missbrauchs einer Ministrantin durch das Amtsgericht Bad Kissingen Rechtsmittel eingelegt, siehe https://www.br.de/nachrichten/bayern/bkindesmissbrauch-fall-gegen-priester-musserneut-vor-gericht,S8qu7Pa.

$10 \mathrm{Zu}$ finden unter https://weltanschauungsrecht.de/sites/default/files/download/ifw_strafanzeige_katholische_kirche.pdf, zuletzt aufgerufen am 26.11.2020.

11 Zit. n. https://www.deutschlandfunkkultur.de/ein-jahr-nach-missbrauchsstudie-vieletaeter-wenig.976.de.html?dram:article_id=459437.

12 Staatsanwaltschaften in Bayern: 204 noch lebende Beschuldigte, bis auf eine noch laufende Ermittlung wurden alle Fälle eingestellt bzw. die Ermittlungen gar nicht erst aufgenommen, siehe https://www.katholisch.de/artikel/26444-nach-mhg-studie-nurnoch-ein-verfahren-gegen-kleriker-in-bayern; Staatsanwaltschaft Freiburg: $5^{1}$ noch lebende Beschuldigte, 5o Fälle wurden eingestellt, ein Verfahren läuft noch, siehe https://staatsanwaltschaft-freiburg.justiz-bw.de/pb/,Lde/630o861; Staatsanwaltschaft Köln: 9 Beschuldigte, die nicht bereits verurteilt wurden, alle Verfahren wurden eingestellt, siehe https://www.deutschlandfunkkultur.de/ein-jahr-nach-missbrauchsstudie-vieletaeter-wenig.976.de.html?dram:article_id=459437 (Fn. 11); Staatsanwaltschaft Limburg: Ermittlungen wurden nicht aufgenommen, allerdings zum Teil auch unter Berufung auf den Einstellungsgrund der Verjährung, siehe https://www.sueddeutsche.de/panorama/ justiz-limburg-an-der-lahn-staatsanwaltschaft-limburg-keine-ermittlungen-zumissbrauch-dpa.urn-newsml-dpa-com-20090101-190515-99-230814; Staatsanwaltschaften Hamburg, Schleswig-Holstein, Mecklenburg-Vorpommern: 33 Beschuldigte, bis auf eine noch laufende Ermittlung wurden alle Fälle eingestellt bzw. die Ermittlungen gar nicht erst aufgenommen, siehe https://www.domradio.de/themen/bistuemer/202O-04-27/ ermittlungen-meist-eingestellt-missbrauchsfaelle-im-erzbistum-hamburg. Die Generalstaatsanwaltschaft Koblenz hat zunächst keine Ermittlungen aufgenommen, jedoch ist es später zu Ermittlungen der Staatsanwaltschaften in Rheinland-Pfalz gekommen: 91 Fälle, bis auf eine noch laufende Ermittlung wurden alle Fälle eingestellt bzw. die Ermittlungen 


\section{Ermittlungstätigkeiten deutscher Staatsanwaltschaften}

Die Ermittlungsarbeit deutscher Staatsanwaltschaften im Hinblick auf Fälle des sexuellen Missbrauchs von Kindern innerhalb der katholischen Kirche hat mit mehreren Schwierigkeiten zu kämpfen. Häufig fehlt es an Strafanzeigen. Dies erschwert die Ermittlung der Identität von Täter und Opfer sowie der notwendigen Angaben über Ort und Zeit der jeweiligen Tat. Die MHG-Studie kann für sich genommen diese Erkenntnisdefizite nicht beheben, da sie weitgehend auf der Basis anonymisierter Akten erfolgt ist. ${ }^{13}$ Hinzu tritt, dass viele Taten so weit zurückliegen, dass bereits Verjährungsfristen wirksam geworden sind. Dies steht der Strafverfolgung entgegen. Nicht zuletzt ist zu berücksichtigen, dass nicht alle der in der MHG-Studie als Missbrauch gekennzeichneten Fälle auch eine Straftat darstellen.

Gleichwohl wurden etwa in Bayern Ermittlungen aufgenommen, die zunächst 204 katholische Kleriker betrafen. Die bislang eingeleiteten Ermittlungen wurden allerdings bis auf einen Fall zwischenzeitlich eingestellt. Die Gründe hierfür sind vielschichtig und betreffen insbesondere die bereits genannte Verjährungsproblematik sowie den Umstand, dass bestimmte Taten nicht die Anforderungen der gesetzlichen Straftatbestände erfüllen. Teilweise handelte es sich außerdem um Fälle, in denen es in der Vergangenheit schon zu einer Verurteilung gekommen ist. Von Interesse wären freilich die Gründe dafür, weshalb in 49 Verfahren, wie das bayerische Justizministerium mitteilt, keine zureichenden Anhaltspunkte für eine Straftat oder kein hinreichender Tatverdacht vorlagen. Weil diese nicht bekannt sind, kann hierzu nicht Stellung genommen werden.

Auch in anderen Staatsanwaltschaften sieht es Medienberichten zufolge kaum anders aus. ${ }^{14}$ Nähere Kenntnis besteht zumindest in Bezug auf die Positionierung der Generalstaatsanwaltschaft Koblenz. ${ }^{15}$ Diese lehnte die Aufnahme von Ermittlungen in Bezug auf Taten, die mutmaßlich in ihrem Zuständigkeitsbereich stattgefunden haben (immerhin vier Bistümer), per se

gar nicht erst aufgenommen, siehe das Schreiben des rheinland-pfälzischen Ministeriums der Justiz an den Präsidenten des Landtags Rheinland-Pfalz vom 15.5.2019, einzusehen unter http://dokumente.landtag.rlp.de/landtag/vorlagen/4817-V-17.pdf, 4ff. sowie https://www.sueddeutsche.de/panorama/justiz-mainz-bistuemer-listen-zu-sexuellemmissbrauch-fast-abgearbeitet-dpa.urn-newsml-dpa-com-2oogo1o1-2002o6-99-795479.

13 Dreßing u.a., MHG-Studie (Fn. 7), 3.

14 Zur Übersicht der Ermittlungstätigkeit siehe Fn. 12.

15 Ebenso verfügte die Staatsanwaltschaft Frankenthal, vgl. das Schreiben des rheinlandpfälzischen Ministeriums der Justiz an den Präsidenten des Landtags Rheinland-Pfalz vom 15.5.2019 (Fn. 12), 3 . 
ab. ${ }^{16}$ Als Grund wird unter Bezugnahme auf die MHG-Studie sowie die Strafanzeige der Strafrechtsprofessoren vorgebracht: „Weder aus der Strafanzeige noch aus der Studie selbst ergeben sich zureichende tatsächliche Hinweise auf eine nach Tatzeit, Tatort, betroffener und handelnder Person sowie Tathandlung eingrenzbare verfolgbare Straftat. ${ }^{{ }^{17}}$ Die Generalstaatsanwaltschaft Koblenz ist weiter der Ansicht, ihre Ermittlungspflicht erstrecke sich "nicht auf das Aufspüren verdächtiger Personen“, weshalb es dieser insbesondere entgegenstünde, dass die Studie keine persönlichen Angaben zur Identifikation von Tätern enthält. ${ }^{18}$

\section{Begründung eines Anfangsverdachts durch die MHG-Studie}

Die Position der Generalstaatsanwaltschaft Koblenz ist aus rechtswissenschaftlicher Sicht nicht haltbar. Die in der MHG-Studie enthaltenen Informationen begründen durchaus einen hinreichenden Tatverdacht, wie er für die Aufnahme von staatsanwaltschaftlichen Ermittlungen erforderlich ist. Durch pauschale Ablehnung ihrer Ermittlungspflicht hat die Generalstaatsanwaltschaft Koblenz gegen das Legalitätsprinzip verstoßen. Im Einzelnen:

In $\S 160$ Abs. 1 der Strafprozessordung (StPO) heißt es: „Sobald die Staatsanwaltschaft durch eine Anzeige oder auf anderem Wege von dem Verdacht einer Straftat Kenntnis erhält, hat sie zu ihrer Entschließung darüber, ob die öffentliche Klage zu erheben ist, den Sachverhalt zu erforschen." Die Vorschrift trägt damit dem das deutsche Strafverfahren leitenden Legalitätsgrundsatz Rechnung, wonach eine staatliche Verpflichtung besteht, Straftaten durch eigene Behörden zu verfolgen. ${ }^{19}$ Diese Pflicht ist die zweite Seite einer Medaille, auf deren erster Seite sich ein Verbot von Privatfehden zeigt. ${ }^{20}$

16 Zumindest wurden aber zur "strafrechtliche(n) Aufarbeitung“ Gespräche mit den in ihren Zuständigkeitsbereich fallenden Bistümern geführt und die auf dieser Basis erlangten Informationen auf ihre strafrechtliche Relevanz überprüft. Zu einem Zwischenergebnis siehe ebd., 4.

17 Siehe zum Zitat Scheinfeld/Willenbacher, Neue Juristische Wochenschrift 2019, 136of., die auf das Schreiben der GenStA Koblenz an das Institut für Weltanschauungsrecht (ifw) vom 6.2.2019 verweisen.

18 Zu einer ähnlichen Rechtseinschätzung siehe die Äußerung der niedersächsischen Justizministerin Barbara Havliza, einzusehen unter https://www.lto.de/recht/nachrichten/n/ missbrauch-skandal-katholische-kirche-strafanzeige-strafrecht-professoren.

19 Vgl. Münchener Kommentar StPO/Kölbel, 1. Aufl. 2016, § 16o Rn. 2; zum Legalitätsprinzip Münchener Kommentar StPO/Peters, 1. Aufl. 2016, § $15^{2}$ Rn. $26 \mathrm{ff}$.

20 Vgl. Münchener Kommentar StPO/Peters (Fn. 19), § $15^{2}$ Rn. 2ff. Zur materiellen Absicherung der staatlichen Verfolgungspflicht durch § 339 StGB (Rechtsbeugung) 
Insbesondere zur erfolgreichen Herstellung von Rechtsfrieden ist das Fehderecht dem Bürger genommen. Ereignen sich Straftaten, wird der Umgang damit dem Staat überantwortet, der insbesondere aufgrund seiner Neutralität friedensstiftend wirken soll. Die Aufnahme eines Strafverfahrens liegt daher grundsätzlich nicht im Ermessen der Staatsanwaltschaften, sondern ist ihre originäre, rechtliche Pflicht. ${ }^{21}$

Zur Voraussetzung für die Aufnahme von Ermittlungen erhebt das Gesetz den „Verdacht einer Straftat“. Näher konkretisiert wird dies noch von $§ 152$ Abs. 2 StPO, wonach die Staatsanwaltschaft "soweit nicht gesetzlich ein anderes bestimmt ist, verpflichtet (ist), wegen aller verfolgbaren Straftaten einzuschreiten, sofern zureichende tatsächliche Anhaltspunkte vorliegen." Abgeleitet wird aus der Norm das Erfordernis eines Anfangsverdachts zur Aufnahme staatsanwaltschaftlicher Ermittlungen. Ein solcher liegt vor, wenn es nach kriminalistischer Erfahrung möglich erscheint, dass eine verfolgbare Straftat begangen worden ist. ${ }^{22}$ Es muss also die Möglichkeit einer späteren Verurteilung bestehen. ${ }^{23}$ Sinn und Zweck des Anfangsverdachts ist es, den Bürger vor unnötigen, unbegründeten und unangemessenen staatlichen Übergriffen, insbesondere Willkür zu schützen. Um zugleich dem staatlichen Interesse an Strafverfolgung hinreichend Rechnung zu tragen, sind die Anforderungen an den Anfangsverdacht indes als verhältnismäßig gering einzustufen. ${ }^{24}$

Einem Anfangsverdacht steht das Fehlen genauer Angaben zu Tatort und Tatzeit ${ }^{25}$ oder zur Identität des Täters ${ }^{26}$ nicht entgegen. Diese Informationen zu erzielen, ist gerade Gegenstand des Ermittlungsverfahrens. Aus diesem Grund kann etwa auch ein Strafverfahren gegen Unbekannt geführt werden. Freilich darf sich die Staatsanwaltschaft nicht in Vermutungen ergehen, sondern muss

siehe Leipziger Kommentar StGB/Hilgendorf, 12. Aufl. 20o9, § 339 Rn. 20; Münchener Kommentar StGB/Uebele, 3. Aufl. 2019, § 339 Rn. 12; Systematischer Kommentar StGB/ Stein/Deiters, 9. Aufl. 2016, § 339 Rn. 3 o.

21 Ausnahmen von der Pflicht zur Strafverfolgung können sich aus in den $§ \S 153 \mathrm{ff}$. StPO geregelten Opportunitätsgründen ergeben. Zum „Opportunitätsprinzip“ siehe Münchener Kommentar StGB/Peters (Fn. 19), § $15^{2}$ Rn. 75ff.; Systematischer Kommentar StPO/Weßlau/Deiters, 5. Aufl. 2016, Vor. §§ 151 ff. Rn. $7 \mathrm{ff}$.

22 Vgl. BGH, Neue Juristische Wochenschrift 1989, 96 und 97; Karlsruher Kommentar StPO/Diemer, 8. Aufl. 2019, § 152 Rn. 7; Meyer-Goßner/Schmitt/Schmitt, 63. Aufl. 2020, StPO $§ 152$ Rn. 4.

23 Münchener Kommentar StPO/Peters (Fn. 19), § 152 Rn. 35.

24 Vgl. Karlsruher Kommentar StPO/Diemer (Fn. 22), § 152 Rn. 7; Münchener Kommentar StPO/Peters (Fn. 19), § $15^{2}$ Rn. 36; Systematischer Kommentar StPO/Weßlau/Deiters (Fn. 21), § 152 Rn. 12d.

25 Vgl. Scheinfeld/Willenbacher, Neue Juristische Wochenschrift 2019, 1357 und 1358.

26 Vgl. Münchener Kommentar StPO/Peters (Fn. 19), §152 Rn. 43f.; Löwe-Rosenberg/Mavany, 27. Aufl. 2020, StPO, § 152 Rn. 3 o. 
ihre Einschätzung auf konkrete Tatsachen stützen. ${ }^{27}$ Eine solche Tatsache kann auch in einem nicht völlig haltlosen Gerücht oder einer Behauptung Dritter begründet liegen, denn die Prüfung des Wahrheitsgehalts ist gerade die Aufgabenstellung für das Ermittlungsverfahren. ${ }^{28}$ Auch das Fehlen der Kenntnis der Opferidentität ist für die Aufnahme staatsanwaltschaftlicher Ermittlungen unschädlich. ${ }^{29}$ Ein Anfangsverdacht besteht vielmehr bereits dann, wenn es wahrscheinlich ist, dass es eine Straftat, ${ }^{30}$ einen Täter und (sofern das jeweilige Delikt dies voraussetzt) ein Opfer gibt. Anderenfalls könnte zum Beispiel eine Vielzahl von Internetstraftaten nicht verfolgt werden, bei denen nicht selten zunächst Hinweise auf ein kriminelles Verhalten auffallen, ohne dass die Identität der Täter oder der betroffenen Opfer auch nur im Ansatz geklärt ist.

Die in der MHG-Studie mitgeteilten Informationen zu Missbrauchsfällen in der katholischen Kirche begründen einen Anfangsverdacht im dargelegten Sinne. ${ }^{31}$ Soweit es darin um Übergriffe auf Opfer unter 14 Jahren geht, die teils sogar das orale, anale oder vaginale Eindringen umfassen, handelt es sich hierbei nach derzeitiger Rechtslage ${ }^{32}$ um Straftaten des sexuellen bzw. des schweren sexuellen Missbrauchs von Kindern, §§ 176, 176a Abs. 2 Strafgesetzbuch (StGB). Der Annahme eines Anfangsverdachts steht es nicht entgegen, dass die Daten nicht in den Originalakten erhoben wurden, sondern seitens der Kirche in anonymisierter Form an die Wissenschaftler überreicht wurden. Der kriminalistischen Erfahrung widerspricht es, anzunehmen, die Kirche würde Missbrauchsfälle mitteilen, die sich real nicht in den Akten finden. Für die katholische Kirche bedeutet die Transparentmachung von sexuellem Missbrauch durch ihre Kleriker das Risiko eines Reputationsschadens, der gar den Austritt einer Vielzahl von Mitgliedern zur Folge haben kann - und dies auch

27 Vgl. Bundesverfassungsgericht, Neue Juristische Wochenschrift 2014, 3085 und 3087; Löwe-Rosenberg/Mavany (Fn. 26), § 152, Rn. 28; Münchener Kommentar StPO/Peters (Fn. 19) § 152, Rn. 42 sowie zur schweizerischen Strafprozessordnung Walder, Zeitschrift für die gesamte Strafrechtswissenschaft 95, 1983, hier 862 und $867 \mathrm{ff}$.

28 Vgl. Löwe-Rosenberg/Mavany (Fn. 26) § 152, Rn. 30, 32.

29 Vgl. Scheinfeld/Willenbacher, Neue Juristische Wochenschrift 2019, 1357f.

30 Zur Debatte, ob auch legales Verhalten einen Anfangsverdacht begründen kann, siehe Löwe-Rosenberg/Mavany (Fn. 26) § 152 Rn. 36; zu dieser Frage speziell im Fall Edathy siehe Bundesverfassungsgericht, Neue Juristische Wochenschrift 2014, 3085 und $3087 \mathrm{f}$. Kritisch zur Entscheidung Hoven, Neue Zeitschrift für Strafrecht 2014, 361.

$31 \mathrm{Zu}$ dieser Einschätzung gelangen auch Scheinfeld/Willenbacher, Neue Juristische Wochenschrift 2019, 1357; Putzke/Hilgendorf/Herzberg/Merkel/Neumann/Rössner, Strafanzeige (Fn. 10).

32 Siehe schon oben (Fn. 2) dazu, dass in näherer Zukunft mit einer Gesetzesreform zu rechnen ist. 
hatte. ${ }^{33}$ Es liegt daher fern, dass gegenüber den Wissenschaftlern Fehlangaben getätigt wurden.

Gegen die Annahme eines Anfangsverdachts spricht es auch nicht, dass ein Teil - gegebenenfalls sogar der größte Teil - der in der Studie genannten Taten bereits der Verjährung unterliegt und damit von staatlicher Seite nicht mehr verfolgbar ist. Der sexuelle Missbrauch an Kindern gemäß § 176 StGB verjährt in zehn Jahren, $\S 78 \mathrm{Abs}$. $3 \mathrm{Nr}$. 3 StGB, bzw. nach Umsetzung der Gesetzesreform zu den Straftaten der sexuellen Gewalt gegen Kinder, nach 20 Jahren, § 78 Abs. 3 Nr. 2 StGB. Letzteres gilt schon nach der geltenden Rechtslage für den schweren sexuellen Missbrauch von Kindern. Dabei ist zu berücksichtigten, dass die Verjährungsfristen der hier in Rede stehenden Straftaten seit 2015 erst mit Vollendung des 30 . Lebensjahrs des Opfers zu laufen beginnen. ${ }^{34}$ Weil die Studie den Zeitraum von 1946 bis 2014 umfasst, besteht daher durchaus noch die Wahrscheinlichkeit, dass sich nicht verjährte, strafrechtlich relevante Taten ereignet haben, auf die die Studie hinweist.

Die Generalstaatsanwaltschaft Koblenz ist aus rechtswissenschaftlicher Sicht vor diesem Hintergrund ihrem staatlichen Ermittlungsauftrag zu Unrecht nicht nachgekommen. Hierin liegt ein Verstoß gegen das Legalitätsprinzip, der ernstzunehmende Folgen in Bezug auf das Vertrauen in die staatlichen Organe und das Funktionieren des Gemeinwesens haben kann.

\section{Blinde Justitia?}

Abgesehen von der Positionierung der Generalstaatsanwaltschaft Koblenz sind keine vergleichbaren, eklatanten Verletzungen des Legalitätsprinzips vonseiten deutscher Staatsanwaltschaften bekannt. In den meisten Bundesländern wurde ermittelt und dann im Anschluss an eine Prüfung der Aktenlage eingestellt bzw. in Einzelfällen wurden weitere Verfahrensschritte wie Anklagen eingeleitet. ${ }^{35}$ Dennoch stellt sich die Frage, ob die Ermittler durch ihr Verhalten den Anforderungen gerecht geworden sind, die von Rechts wegen an sie gestellt werden. Zweifel hieran werden geäußert in Bezug auf die Tatsache, dass sämtliche ermittelnden deutschen Strafverfolgungsbehörden ausschließlich in Kooperation mit der katholischen Kirche tätig geworden sind, indem

33 Vgl. https://www.domradio.de/themen/bistümer/2019-o7-21/wenn-die-distanz-waechstexpertin-missbrauch-ist-zurzeit-oft-grund-fuer-kirchenaustritt.

34 49. Gesetz zur Änderung des Strafgesetzbuches vom 21.1.2015, Bundesgesetzblatt Jg. 2015 Teil I Nr. 2, 10 und 11.

35 Zur Übersicht der Ermittlungstätigkeit siehe Fn. 12. 
sie nämlich erforderliche Akten von den jeweiligen Bistümern verlangten, die den Ermittlern auch zur Verfügung gestellt wurden. Durchsuchungen als ein verbreitetes Ermittlungsinstrument von Strafverfolgungsbehörden sind in kirchlichen Räumlichkeiten wie insbesondere den in der MHG-Studie genannten Geheimarchiven nicht erfolgt. ${ }^{36}$ Genügt dies dem Anspruch an die Ermittlungsbehörden oder hätten sie die Augenbinde der Justitia bildlich gesprochen anheben müssen, um einen klareren Blick auf das Verhalten katholischer Kleriker in den letzten Jahren und Jahrzehnten zu erlangen?

Das Bild ist freilich schief: Ihre Binde trägt die Justitia gerade, um sich in ihrer freien Entscheidungsfindung nicht durch spezifische Interessen beeinflussen zu lassen. ${ }^{37}$ Es fragt sich aber, ob die Unterlassung weiterer Ermittlungsmaßnahmen wie zum Beispiel der Durchsuchung bei anderen als der Tat verdächtigen Personen gemäß § 103 StPO zu der Annahme veranlasst, dass der Justitia in Sachen katholische Kirche die Augenbinde bereits zum Nachteil der Gerechtigkeit verrutscht ist. Dafür könnte sprechen, dass immer wieder von unterschiedlichen Seiten die Vermutung geäußert wurde, Akten seien manipuliert worden bzw. es sei in sonstiger Weise vertuscht worden. Der MHG-Studienleiter Dreßing stellte in einer Vergleichsstudie fest, dass in der katholischen Kirche in der Vergangenheit viel häufiger sexualisierte Gewalt gegen Kinder und Jugendliche vertuscht wurde als in anderen Institutionen. ${ }^{38}$ Entsprechende Aussagen zur Vertuschung von Missbrauchstaten innerhalb der katholischen Kirche lassen sich auch der MHG-Studie selbst an verschiedenen Stellen entnehmen. ${ }^{39}$ Hieraus lässt sich zwar nicht ableiten, dass die für die MHG-Studie bereitgestellten Materialien (die später auch den Staatsanwaltschaften zur Verfügung gestellt wurden) manipuliert wurden. Allerdings liegt nahe, dass diese Unterlagen bereits Ergebnis früherer Vertuschungen sind, denen durch ein darüber hinaus ausgedehntes Aktenstudium weiter hätte nachgegangen werden können. Zwar bestünde insoweit das Risiko, dass relevante Akten zu einem viel früheren Zeitpunkt vernichtet wurden. Ausgeschlossen ist aber nicht, dass sich nicht doch noch strafrechtlich verwertbare Ergebnisse auffinden ließen, die über die Inhalte der seitens der Bistümer freigegebenen Materialien hinausgehen.

36 Vgl. https://www.deutschlandfunkkultur.de/ein-jahr-nach-missbrauchsstudie-vieletaeter-wenig.976.de.html?dram:article_id=459437.

$37 \mathrm{Zu}$ den verbundenen Augen der Justitia siehe Maunz/Dürig/Kirchhof, 91. EL April 2020, Grundgesetz Art. 3 Abs. 1 Rn. 43.

38 Siehe Hermann u.a., Monatsschrift für Kriminologie und Strafrechtsreform, 102, 2019, 249 und $258 \mathrm{f}$.

39 Zur Vertuschung im Vergleich zu anderen Institutionen siehe Dreßing u.a., MHG-Studie (Fn. 7), 189ff. Zur Vertuschung allgemein siehe ebd., 124f., 127ff. und $305 \mathrm{ff}$. 
Dabei ist zu berücksichtigen, dass die Durchführung einer Durchsuchung nach $\S 103$ Abs. 1 S. 1 StPO am Grundsatz der Verhältnismäßigkeit zu bemessen ist. ${ }^{40}$ Insofern bedarf es neben dem Anfangsverdacht eines "konkreten Verdacht(s) dafür, dass bestimmte Beweismittel gefunden werden können “41. Es ist zu berücksichtigen, dass bei der Anordnung der Maßnahme die Kooperationsbereitschaft desjenigen, gegen den sich die Durchsuchung richtet, zu berücksichtigen ist. Die Kirche hat ausweislich der Bekundungen verschiedener Ermittlungsbehörden stets kooperiert. Insoweit ist es auch nicht unüblich, in Fällen der Durchsuchung gemäß § 103 StPO auf die Kooperationsbereitschaft des Gegenübers zu setzen, weshalb dies etwa im Kontext von Wirtschaftsstrafverfahren nicht selten praktiziert wird. Gleichwohl gibt es auch in diesem Bereich immer wieder Durchsuchungen, zum Beispiel in Unternehmen, um dort vermutete Informationen über Taten von Unternehmensmitarbeitern zu erlangen. ${ }^{42}$ In diesen Fällen ist allerdings die Schwierigkeit zu berücksichtigen, dass sich den Ermittlern häufig eine Flut an verfügbaren Daten bietet, die nicht ohne Weiteres in angemessener Zeit gesichtet werden können. Im Hinblick auf die katholische Kirche wurde dies von Seiten einzelner Ermittlungsbehörden zu bedenken gegeben: ${ }^{43}$ Die Kirchenarchive sind besonders umfangreich. Es stellt sich vor diesem Hintergrund die Frage, ob überhaupt ohne die Kooperation der Bistümer sinnvolle Ermittlungsarbeit hätte geleistet werden können.

\section{Schluss}

Zur Aufarbeitung von Fällen des sexuellen Missbrauchs durch katholische Kleriker konnte die Strafjustiz in der Vergangenheit durch punktuelle Verurteilungen ihren Beitrag leisten. Im Anschluss an die MHG-Studie sind die meisten deutschen Staatsanwaltschaften zu Ermittlungstätigkeiten übergegangen, die indes zumeist in Einstellungen endeten. Ein Ausweis für eine blinde Justitia ist hierin aber nicht per se zu sehen. Zwar kann dieser Vorwurf gegenüber einzelnen Vertretern der Strafjustiz erhoben werden, wie das Beispiel der Generalstaatsanwaltschaft Koblenz zeigt. Aus dem bloßen Umstand, dass sich in der Ermittlungsarbeit anderer Behörden beschränkt wurde auf

40 Bundesverfassungsgericht, Neue Juristische Wochenschrift 2007, 1804 und 1805; LöweRosenberg/Tsambikakis, 27. Aufl. 2019, StPO § 103 Rn. 1, 7 .

41 Ebd., § 103 Rn. 2.

42 Vgl. https://www.tagesschau.de/wirtschaft/razzia-deutsche-bank-101.html.

43 Vgl. https://www.deutschlandfunkkultur.de/ein-jahr-nach-missbrauchsstudie-vieletaeter-wenig.976.de.html?dram:article_id=459437. 
die seitens der Bistümer zugänglich gemachten Akten, kann allerdings kein pauschales Urteil im Sinne einer gegenüber der Kirche prinzipiell milde gestimmten Justiz abgeleitet werden. Vielmehr kommt hierin nicht zuletzt der Umstand zum Ausdruck, dass weitergehende Ermittlungsmaßnahmen mit erheblichen Schwierigkeiten behaftet wären. Es wäre insofern im Einzelfall zu prüfen, ob die seitens der Bistümer zur Verfügung gestellten Materialien Anhaltspunkte liefern, die Ermittlungsarbeit darüber hinaus - etwa im Wege von Durchsuchungen in Räumlichkeiten der Kirche - ausdehnen zu müssen. 